\title{
Arbuscular mycorrhizal fungi associated with shade trees and Coffea arabica L. in a coffee-based agroforestry system in Bonga, Southwestern Ethiopia
}

\author{
Tadesse Chanie Sewnet (1) \& Fassil Assefa Tuju (2)
}

(I) Haramaya University, Ethiopia

(2) Addis Ababa University, Ethiopia

In a first step to understand the interactions between Coffea arabica L. trees and mycorrhizae in Ethiopia, an investigation of the current mycorrhizal colonization status of roots was undertaken. We sampled i4 shade tree species occurring in coffee populations in Bonga forest, Ethiopia. Milletia ferruginea, Schefflera abyssinica, Croton macrostachyus, Ficus vasta, F. sur, Albizia gummifera, Olea capensis, Cordia africana, Ehretia abyssinica, Pouteria adolfi-friederici, Pavetta oliveriana, Prunus africana, Phoenix reclinata and Polyscias fulva. Coffee trees sampled under each shade tree were all shown to be colonized by arbuscular mycorrhizal fungi (AM fungi). Four genera and 9 different species of AM fungi were found in the soils. Glomus (Sp1, Sp2, \& Sp3 \& Sp4), Scutellospora (Sp1 \& Sp2) and Gigaspora (Sp1 \& Sp2) were found under all 14 shade tree species, whereas Acaulospora (Sp1) occurred only in slightly acidic soils, within a pH range of 4-93-5-75. Generally, roots of the coffee trees were colonized by arbuscules to a greater degree than those of their shade trees, the arbuscular colonization percentage (AC\%) of the former being higher than the latter (significant difference at 0.05 level). Though differences were not statistically significant, the overall hyphal colonization percentage ( $\mathrm{HC} \%$ ) and mycorrhizal hyphal colonization percentage (MHC\%) were shown to be slightly higher under coffee trees than under their shade trees. However, the differences were statistically significant at 0.05 level in the case of $\mathrm{HC} \%$ values of coffee trees under Pouteria adolf-friederici and MHC\% under Cordia africana. Spore density and all types of proportional root colonization parameters ( $\mathrm{HC} \%, \mathrm{MHC} \%, \mathrm{AC} \%$ and vesicular colonization percentage, $\mathrm{VC} \%$ ) for both coffee and shade trees were negatively and significantly correlated with organic soil carbon, total $\mathrm{N}$, available $\mathrm{P}, \mathrm{EC}$ and $\mathrm{Zn}$. Correlation between arbuscular colonization for coffee ( $\mathrm{AC} \%)$ and organic carbon was not significantly positive at a 0.05 level. Incidence of specific spore morphotypes was also correlated with physical and chemical soil properties. Results indicate that AM fungi could potentially be important in aforestation and help to promote coffee production activities in Ethiopia providing an alternative to expensive chemical fertilizer use, and would offer management methods that take advantage of natural systems dynamics that could potentially preserve and enhance coffee production.

Key words: aforestation; agroforestry, coffee groves, ecology, integrated production systems, rhizosphere, sustainable agriculture, symbiosis 


\section{Introduction}

Forests are important gene reservoirs and natural regeneration areas for many economically important plants around the world (Wolf, I999; Daba, 2002). Coffea arabica L., or arabica coffee, is a non-alcoholic stimulant beverage crop and one of the most valued crops in world trade. It has for many years remained second in value only to oil as a source of foreign exchange in numerous developing countries (Tefestewolde, I995; ICO, 2007). Ethiopia is believed to be the country of origin of arabica coffee (Paulose and Demel, 2000). It represents over $70 \%$ of the world's coffee production (ICO, 2009). In 2002, it contributed to more than $60 \%$ of Ethiopia's foreign exchange earnings, over $5 \%$ of GDP, I $2 \%$ of agricultural output, and $10 \%$ of government revenues (CSA, 2002). According to somewhat older data, it also employs circa $25 \%$ of domestic labour force (EIAR, I996). About $55 \%$ of the country's production is exported while the balance is consumed locally (Mesfin, I99I). In Ethiopia, coffee grows in natural coffee groves and managed agroforestry systems, totaling about 500,000 hectares (Aga et al., 2003).

More than $60 \%$ of coffee plants grow under shade trees in evergreen forest areas situated in southwestern Ethiopia (Paulose and Zebene, 1994). Shade tree genera include Albizia, Acacia, Bersama, Cordia, Croton, Dracaena, Entada, Erythrina, Ficus, Leucaena, Millettia, and Syzygium (FAO, I968; Demel and Tigeneh, I99I). Like many crops, coffee and shade trees associate symbiotically with arbuscular mycorrhizal fungi (AM fungi) (Sieverding, I991; Cardoso, 2003; Muleta et al., 2007).

Mycorrhizae are thought to influence plant community composition and plant productivity (Van der Heijden et al., I998). Moreover, success of any reforestation intervention is likely to depend on the co-establishment of diverse AM fungi together with seedlings in the nursery (Sieverding, I991; Francis and Read, 1994). Benefits from mycorrhizae are greatest and most obvious under low input subsistence agriculture systems in developing countries in the tropics (Sieverding, I99r).

Propagules of mycorrhizae in coffee soils enhance coffee plant growth, increase $P$ and $\mathrm{Zn}$ uptake of young coffee seedlings in nursery conditions and improve their establishment after transplantation (Lopes et al., 1985; Rivera et al., 2003; Vaast and Zasoski, 1992). They also impart tolerance against a number of plant parasitic nematodes (Vaast et al., I998). These positive effects of mycorrhizal fungi could have significant importance for low input agriculture (Douds et al., 2000).

A number of recent studies have described the relationship of mycorrhizal symbiosis with Acacia polyacantha obtained from a dry savannah woodland ecosystem (Yonase, 2005), and Erythrina brucei from a highland woodland ecosystem (Shasho, 2002). Mycorrhizae (esp. Glomus spp.) were also shown to colonize tamarind roots in different ecological zones in Senegal, which is a country with an essentially dry climate. Inoculation of young seedlings helped to generate increased drought tolerance and can thus be considered an active tool in tamarind management (Bourou et al., 2010 \& 20II). Mycorrhizal colonization rates of a number of tree species in an afromontane forest were also estimated (Tesfaye et al., 2003a). On top of that, the number of coffee shade tree species and density of AM fungi spores in Bonga natural coffee forest soils of southwestern Ethiopia 
(in the same forest as the present study but at different locations) were also investigated (Muleta et al., 2007). The latter authors identified the dominant coffee shade tree species, evaluated their densities, and quantified and characterized AM fungi populations particularly within the rhizosphere of coffee plants. In Brazil, Cardoso et al. (2003) indicated that greater numbers of spores in the deeper soil layers of agroforestry and coffee stands may be due to greater amounts of roots at those depths. Additionally, the same authors explained that greater mycorrhizal incidence at deeper soil layers in the agroforestry system may change the dynamics of phosphorus cycling in soil, making this nutrient more available to plants. In Ethiopia there is almost no information available on the interrelationship between diversity of AM fungi, their density, root colonization rate (RCR) and the nutrient dynamics of coffee and their shade trees.

The present study was therefore initiated with the following objectives, to (a) quantify and identify spores of AM fungi occurring under the different shade tree species, and (b) investigate the relationship between AM fungi, root colonization, and physical and chemical soil parameters in a number of natural coffee forest sites in Bonga, Ethiopia, where some natural forest relics are found.

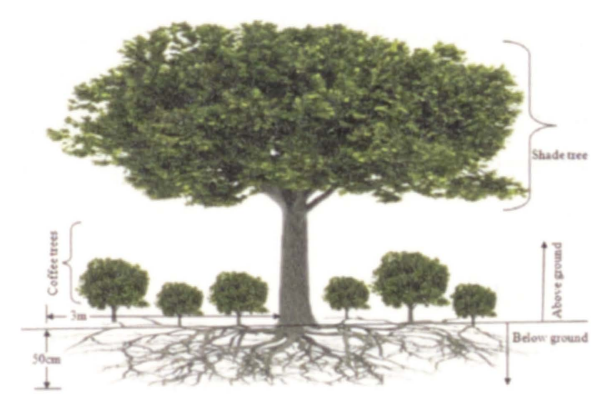

Figure 1: A sketch of shade tree and understory coffee plants 


\section{Materials and methods}

Study site

This study was carried out in Bonga coffee forest situated $15 \mathrm{~km}$ from Bonga town, Ethiopia (Fig. 2).

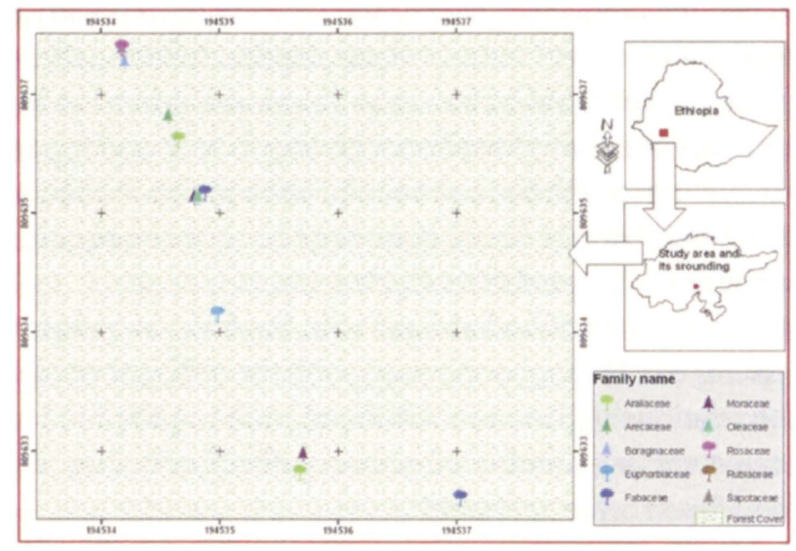

Figure 2: GPS coordinates of sample shade tree species in the study area (coordinates of shade tree species in the figure were taken from table 1). Source: Adopted from Ethiopian Map Agency, digitized and modified using GIS technology

The forest was identified and demarcated by the government of Ethiopia as a coffee forest and forest genetic reservoir for conservation (MOA, I998). The site is one of the national coffee forest areas defined in Southern Nations and Nationalities People's Regional State (SNNPRS) of the country. Its altitude ranges 1750-2000 masl. Average annual rainfall amounts to over $1700 \mathrm{~mm}$. Mean annual temperature ranges between $18-20^{\circ} \mathrm{C}$, with mean daily temperature minima of $8.7^{\circ} \mathrm{C}$ and corresponding mean maxima of $29.9^{\circ} \mathrm{C}$ (Taye and Burkhard, 20Ir).

\section{Sampling roots and $A M$ fungi}

Point locations of 14 dominant shade tree species were determined using Garmin 12 Handheld GPS Receiver (Table I). Root samples of the shade trees and their associated coffee trees ( 3 coffee plants under each shade tree) were collected by excavating soil using a handheld hoe starting from the shade tree's trunk base and working out towards the fine roots within a $3 \mathrm{~m}$ radius. Soil samples taken at $50 \mathrm{~cm}$ depth were washed over 2 (to restrain bigger roots) and $0.5 \mathrm{~mm}$ sieves. Per sample, twenty fine roots were randomly collected from the $0.5 \mathrm{~mm}$ sieve and brought into the laboratory, carefully washed with tap water, cut into $\mathrm{I} \mathrm{cm}$ pieces and placed in 50\% alcohol until processed (Frioni et al., 1999). The area under each tree canopy was partitioned into six sectors of 600 each. A total of $600 \mathrm{~g}$ of triplicates sample (each replica having $200 \mathrm{~g}$ composite soil) was collected at $50 \mathrm{~cm}$ depth from under the canopy of each tree and brought to the laboratory for spore extraction. 


\begin{tabular}{|c|c|c|}
\hline Scientific name & Family name & Location \\
\hline Milletia ferruginea & Fabaceae & $\begin{array}{l}07019^{\prime} \cdot 376^{\prime \prime} \mathrm{N} \\
036014^{\prime} .9477^{\prime \prime} \mathrm{E}\end{array}$ \\
\hline Schefflera abyssinica & Araliaceae & $\begin{array}{l}07019 \cdot 383^{\prime \prime} \mathrm{N} \\
036014 \text { '.899"E }\end{array}$ \\
\hline Croton macrostachyus & Euphorbiaceae & $\begin{array}{l}07019 ' .430 " \mathrm{~N} \\
036014 \text { '.874"E }\end{array}$ \\
\hline Ficus vasta & Moraceae & $\begin{array}{l}07019 \cdot 389 \text { "'N } \\
036014 \text { '.900"'E }\end{array}$ \\
\hline Ficus sur & Moraceae & $\begin{array}{l}07019 \text { '.465"N } \\
036014 \text { '.867" E }\end{array}$ \\
\hline Albizia gummifera & Fabaceae & $\begin{array}{l}07019 ' .466 \text { "N } \\
036014 \text { '.870"'E }\end{array}$ \\
\hline Olea capensis & Oleaceae & $\begin{array}{l}07019^{\prime} \cdot 465^{\prime \prime} \mathrm{N} \\
036014 \text { '.868"'E }\end{array}$ \\
\hline Cordia africana & Boraginaceae & $\begin{array}{l}\text { 07019'.505"N } \\
036014 \text { '.846"E }\end{array}$ \\
\hline Ehretia abyssinica & Boraginaceae & $\begin{array}{l}07019 ' .509 " \mathrm{~N} \\
036014 \text { '.845"E }\end{array}$ \\
\hline Pouteria adolfi-friederici & Sapotaceae & $\begin{array}{l}07019 \text { '.509"N } \\
036014 \text { '.845"E }\end{array}$ \\
\hline Pavetta oliveriana & Rubiaceae & $\begin{array}{l}07019 ' .509 " \mathrm{~N} \\
036014.845^{\prime \prime} \mathrm{E}\end{array}$ \\
\hline Prunus africana & Rosaceae & $\begin{array}{l}07019 ' .509 " \mathrm{~N} \\
036014 ' .845^{\prime \prime} \mathrm{E}\end{array}$ \\
\hline Phoenix reclinata & Arecaceae & $\begin{array}{l}07019 ' .489 \text { "N } \\
036014 \text { '.859"E }\end{array}$ \\
\hline Polyscias fulva & Araliaceae & $\begin{array}{l}07019^{\prime} \cdot 482 \text { "N } \\
036014^{\prime} .862 \text { "E }\end{array}$ \\
\hline
\end{tabular}

Table 1: Coffee shade tree species and their respective geographical locations in Bonga coffee forest studied for AMF colonization

AM fungi spores were separated from the soil by the wet-sieving/gradient centrifugation technique (Brundrett et al., 1996). Spores were counted from roo g soil aliquots using an AJ05 model Turret Dissecting Microscope with a magnification of $40 x$. Spores were grouped into genera of different species according to a number of morphological characteristics such as: spore size, shape, colour, wall structure, hyphal attachment (simple, swollen or bulbous) and Melzer's solution reaction (INVAM, 2004; Merryweather, 2004). Permanent slides were prepared for each different spore morphotype with polyvinyl-alcohol and polyvinyl-alcohol plus Melzer's solution (Merryweather, 2004). The diameter of 
spores was measured using a cci2 model camera mounted on an Olympus Bx 51 microscope which was connected to the AnalySIS ${ }^{\circledR}$ Soft Imaging Systems GmbH version 3.2 software program.

Roots were cleared in 10\% $\mathrm{KOH}$ (Kormanik and McGraw, 1982; Brundrett et al., 1994). Dark-pigmented roots were further bleached with $10 \% \mathrm{H}_{2} \mathrm{O}_{2}$ and acidified with $\mathrm{I} \%$ $\mathrm{HCl}$. Cleared roots were stained in trypan blue (0.05\% in I4:I:I lactic acid: glycerol: water). Proportional colonization (colonization of roots by AM fungi) was estimated using the magnified intersection method. A hair line graticule inserted into an eyepiece acted as the line of intersection with each root at $x 200$ magnification under the compound microscope (McGonigle et al., I990). Percentage of root length colonization (\%RLC) was calculated from roo or more intersections for each root sample (around ro fine root pieces per slide). At each intersection, there were six possible mutually exclusive outcomes. The line might intersect at points $\mathrm{p}, \mathrm{q}, \mathrm{r}, \mathrm{s}, \mathrm{t}$ and $\mathrm{u}$ where, "p" represents intersection at no-fungal structures, "q" arbuscules, "r" mycorrhizal vesicles, "s" arbuscules and mycorrhizal vesicles at a time, " $\mathrm{t}$ " mycorrhizal hyphae but no arbuscules or mycorrhizal vesicles, and " $\mathrm{u}$ " hyphae not seen to be connected to arbuscules or mycorrhizal vesicles.

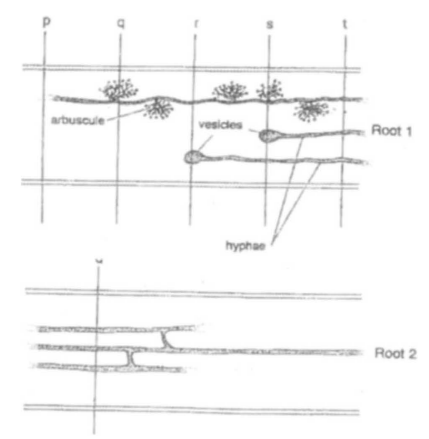

Figure 3: The eyepiece hairline can make 6 types of intersections $(p, q, r, s, t$, and $u)$

Source: Adopted from Practical Methods in Mycorrhizal Research. (1994). Ed. M. Brundrett, L. Melville and L. Peterson

\section{Soil sampling}

A total of $\mathrm{I} \mathrm{kg}$ of composite soil was taken from each sector at $50 \mathrm{~cm}$ depth within $3 \mathrm{~m}$ diameter under each shade tree rhizosphere for physical and chemical soil analyses. Soil samples were analyzed for $\mathrm{pH}$, organic matter (OM), available P (AvP), total N (TN), $\mathrm{Mg}, \mathrm{K}, \mathrm{Ca}, \mathrm{Na}, \mathrm{Zn}$, cation exchange capacity (CEC) and exchangeable cations (EC). Soil $\mathrm{pH}$ was potentiometrically ( $\mathrm{r}$ : I sample) measured by using distilled water (Van Reeuwijk, I993). OM was determined by the wet combustion procedure of Walkley and Black (Van Ranst et al., 1999). TN was determined by the wet or oxidation procedure of the Kjeldahl method (Bremner and Mulvaney, 1982). AvP content of the soil was determined by Olsen method (Van Reeuwijk, I993). CEC and EC of soils were determined by IM ammonium acetate $(\mathrm{pH}$ ) method as presented in the percolation tube procedure (Van Reeuwijk, 
1993). Effective CEC was calculated as the sum of exchangeable cations extracted by ammonium acetate buffered at $\mathrm{pH} 7$ plus $\mathrm{IM} \mathrm{KCl}$ extractable Al. Available $\mathrm{Zn}$ was determined by the diethylenetriaminepentaacetic acid (DTPA) method (Tan, 1996).

\section{Data analysis}

Data were statistically analyzed using the SPSS V.II.o program (SPSS Inc., Chicago, IL., USA). Analysis of variance (ANOVA) was applied to spore numbers and colonization percentages. Means were compared by Duncan's Multiple Range Test at $\mathrm{P} \leq 0.05$.

\section{Results}

AM spores

Four genera and 9 species of AM fungi were identified from the different rhizosphere soils and are presented in table 2 where characteristics exhibited by the different spore types are also indicated. Type I (Glomus spp.) with four different species, type 2 (Gigaspora spp.) with two different species, and type 3 (Scutellospora spp.) with two different species were found under all tree species. Spores of type 4 (Acaulospora spp.), which contains only one species, were found under $64 \%$ of tree species and at lower densities than the first three types (genera) (Tables 2 and 3 ). 


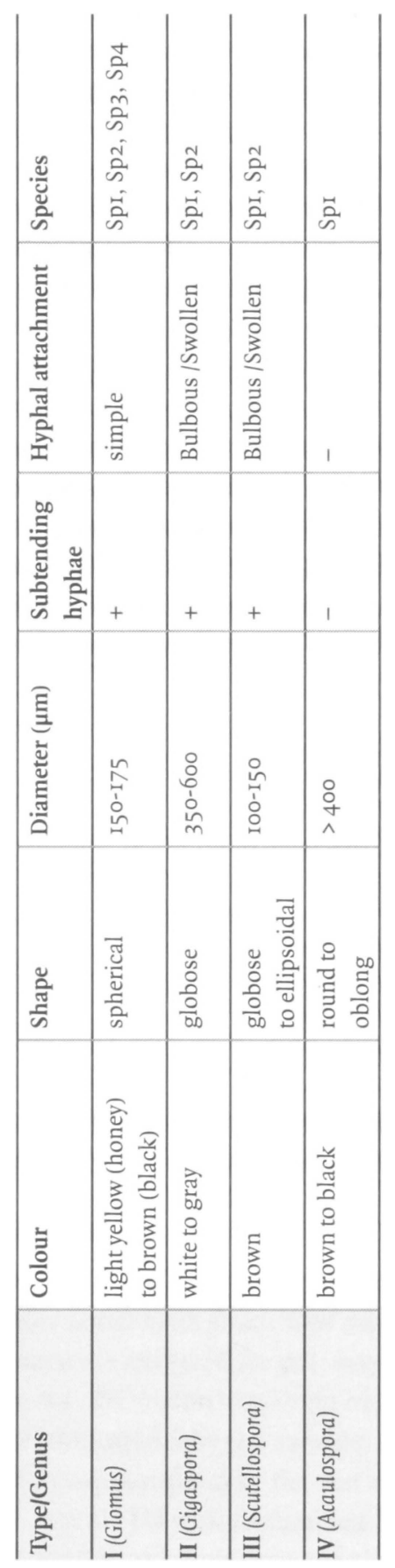

Table 2: Characteristics of Arbuscular Mycorrhizal (AM) fungi spores (based on 15 samples from each type) found in this study. (Where: + = presence of subtending hyphae $/$ - = absence of subtending hyphae (sessile) $/ \mathrm{Sp}=$ species) 
Spore densities recorded in the present study are high, which compares well to other studies. For example, low counts of AMF spores ranging from 4 to 67 spores per $100 \mathrm{~g}$ of dry soil were reported by Muleta et al. (2003) for coffee groves. Similarly, in soils from a dry savannah wood land ecosystem under Acacia polyacantha, Yonase (2005) reported a mean of 57.9 spores per $100 \mathrm{~g}$ of dry soil. Spore densities showed considerable variations across tree species (Table 3). The highest spore density was found under F. vasta, C. macrostachyus and S. abyssinica, the lowest occurred under E. abyssinica, P. africana and P. fulva. Spore density also varied significantly among different members of the same family of shade trees: S. abyssinica and P. fulva in Araliaceae, and F. vasta and F. sur in Moraceae (Table 3).

\begin{tabular}{|c|c|c|c|c|c|}
\hline Species & Total & Type I & Type 2 & Type 3 & Type 4 \\
\hline Milletia ferruginea & $670 \pm 17^{f}$ & $521 \pm 14^{h}$ & $86 \pm 5^{f}$ & $5^{6} \pm 3^{b c}$ & $7 \pm 2^{c}$ \\
\hline Schefflera abyssinica & $997 \pm \mathrm{II}^{\mathrm{c}}$ & $807 \pm 12^{c}$ & $129 \pm 4^{c}$ & $6 \mathrm{I} \pm 4^{\mathrm{bc}}$ & - \\
\hline Croton macrostachyus & $\log 8 \pm 3 I^{b}$ & $892 \pm 15^{b}$ & $149 \pm 8^{b}$ & $57 \pm 2^{b c}$ & - \\
\hline Ficus vasta & $1313 \pm 3^{a}$ & $1089 \pm 17^{a}$ & $17 I \pm 7^{a}$ & $53 \pm \pm^{2 c}$ & - \\
\hline Albizia gummifera & $805 \pm 17^{e}$ & $63 I \pm 5^{f}$ & $106 \pm 2^{d}$ & $63 \pm 2^{a b}$ & $5 \pm 2^{c}$ \\
\hline Olea capensis & $910 \pm 15^{d}$ & $728 \pm 15^{d}$ & I $23 \pm 4^{c}$ & $59 \pm 2^{\mathrm{bc}}$ & - \\
\hline Cordia africana & $760 \pm 17^{e}$ & $57^{6} \pm 10^{g}$ & IOI $\pm 8 \mathrm{~d}^{e}$ & $68 \pm 3^{a}$ & $15 \pm 2^{a b}$ \\
\hline Ehretia abyssinica & $57^{8} \pm \mathrm{II}^{\mathrm{h}}$ & $396 \pm 4^{i}$ & $125 \pm 2^{c}$ & $5^{I} \pm 2^{c}$ & $6 \pm 2^{c}$ \\
\hline Pouteria adolfi-friederici & $787 \pm 13^{e}$ & $623 \pm 8^{f}$ & $93 \pm 3^{\text {ef }}$ & $60 \pm 2^{b}$ & $\mathrm{II} \pm 2^{\mathrm{bc}}$ \\
\hline Pavetta oliveriana & $827 \pm 22^{e}$ & $654 \pm 4^{e}$ & $102 \pm 2^{d}$ & $54 \pm 3^{c}$ & $17 \pm 2^{a}$ \\
\hline Ficus sur & $753 \pm 8^{e}$ & $592 \pm 9^{g}$ & $103 \pm 3^{d}$ & $49 \pm 3^{c}$ & $9 \pm 2^{b c}$ \\
\hline Prunus africana & $635 \pm 9^{g}$ & $483 \pm 9^{i}$ & $96 \pm 3^{e}$ & $50 \pm 3^{c}$ & $6 \pm \mathrm{I}^{\mathrm{c}}$ \\
\hline Phoenix reclinata & $674 \pm 19^{f}$ & $563 \pm 17^{g}$ & $73 \pm 3^{g}$ & $3^{8} \pm 2^{d}$ & - \\
\hline Polyscias fulva & $63^{8} \pm 14^{g}$ & $487 \pm 6^{i}$ & $8 \mathrm{I} \pm 3^{\mathrm{f}}$ & $5^{8} \pm 2 b^{c}$ & $\mathrm{I} 2 \pm 2^{\mathrm{b}}$ \\
\hline
\end{tabular}

Table 3: Spore density per spore type in the rhizosphere of shade trees (spores per $100 \mathrm{~g}$ dry soil, $n=0$ ) Means followed by the same letter in the same column are not significantly different at 0.05 level, \pm s.d.

\section{Root colonization}

All shade tree species were shown to be colonized by mycorrhizae (Table 4). Arbuscules and vesicles were observed in all tree species. General hyphal colonization percentage $(\mathrm{HC} \%)$ varied between members belonging to the same family. While in Fabaceae, colonization of $M$. ferruginia and A. gummifera was statistically similar, it differed in Araliaceae, S. abyssinica ( $76 \%$ ) and P. fulva (56\%), Moraceae, F. vasta $(8 \mathrm{I} \%)$ and F. sur $(65 \%)$ and Boraginaceae, C. africana $(67 \%)$ and E. abyssinica $(52 \%)(\mathrm{P}<0.05)($ Table 4$)$. 


\begin{tabular}{|c|c|c|c|c|}
\hline Species & HC (\%) & MHC (\%) & AC (\%) & VC (\%) \\
\hline Milletia ferruginea & $69 \pm 3^{\mathrm{bc}}$ & $5^{I} \pm 3^{b c}$ & $5 \pm 0.9^{\mathrm{de}}$ & $\mathrm{II} \pm 2^{\mathrm{bc}}$ \\
\hline Schefflera abyssinica & $76 \pm 7^{\mathrm{ab}}$ & $57 \pm 2^{\mathrm{ab}}$ & $6 \pm 0.6^{\mathrm{cd}}$ & $12 \pm 3^{\mathrm{bc}}$ \\
\hline Croton macrostachyus & $8 \mathrm{I} \pm 5^{a}$ & $60 \pm 4^{\text {ab }}$ & $4 \pm 0.5^{e}$ & $14 \pm 0.6^{b}$ \\
\hline Ficus vasta & $8 \mathrm{I} \pm 3^{\mathrm{a}}$ & $62 \pm 4^{a}$ & $3 \pm 0.5^{\mathrm{ef}}$ & $16 \pm 0.6^{a}$ \\
\hline Albizia gummifera & $71 \pm 3^{b}$ & $5 \mathrm{I} \pm 4^{\mathrm{bc}}$ & $6 \pm 0.6^{\mathrm{cd}}$ & $12 \pm 0.6^{b x}$ \\
\hline Olea capensis & $73 \pm 5^{b}$ & $55 \pm 3^{\text {ab }}$ & $5 \pm 0.3^{\mathrm{de}}$ & $12 \pm 0.6^{b c}$ \\
\hline Cordia africana & $67 \pm 3^{\mathrm{bc}}$ & $47 \pm 2^{b c}$ & $7 \pm 0.3^{b}$ & $10 \pm 0 . I^{b c}$ \\
\hline Ehretia abyssinica & $52 \pm 3^{\mathrm{de}}$ & $39 \pm 2^{c}$ & $6 \pm 0.5^{c}$ & $6 \pm 0.3^{d}$ \\
\hline Pouteria adolfi-friederici & $69 \pm 3^{\mathrm{bc}}$ & $52 \pm 3^{b}$ & $5 \pm 0.1^{d}$ & II $\pm 0.6^{b c}$ \\
\hline Pavetta oliveriana & $72 \pm 4^{b}$ & $53 \pm 6^{b c}$ & $6 \pm 0.5^{c}$ & $12 \pm 0.3^{\mathrm{bc}}$ \\
\hline Ficus sur & $65 \pm 4^{\mathrm{bc}}$ & $45 \pm 4^{c}$ & $9 \pm 0.5^{a}$ & $8 \pm 0.3^{d}$ \\
\hline Prunus africana & $54 \pm 3^{d}$ & $40 \pm 3^{c}$ & $3 \pm 0.4^{f}$ & $7 \pm 0.2^{d}$ \\
\hline Phoenix reclinata & $61 \pm 2^{c}$ & $43 \pm 3^{c}$ & $8 \pm 0.5^{\mathrm{ab}}$ & $8 \pm 0.6^{d}$ \\
\hline Polyscias fulva & $5^{6} \pm 4^{\mathrm{cd}}$ & $4^{2} \pm 5^{c}$ & $5 \pm 0.7^{\mathrm{de}}$ & $8 \pm 0.6^{d}$ \\
\hline
\end{tabular}

Table 4: Root colonization percentages of coffee grove shade trees, Bonga, Ethiopia

(Where: $H C$, hyphal colonization $=100[(G-p) / G] ; M H C$, mycorrhizal hyphal colonization $=100[(q+r+s+t) / G] ; A C$, arbuscular colonization $=100(q+s / G) ; V C$, vesicular colonization $=100(r+s / G)$. Where: $G=(p+q+r+s+t+u)$ intersections inspected, $p: n 0$ fungal structures, q: arbuscules, r: mycorrhizal vesides, s: arbuscules and mycorrhizal vesides, t: mycorrhizal hyphae but no arbuscules or mycorrhizal vesides and $u$ : hyphae not seen to be connected to arbuscules or mycorrhizal vesides).

Percentages of coffee roots colonized by total hyphae (HC\%) were generally higher than for the respective associated shade tree species. Mycorrhizal hyphal colonization (MHC\%) of coffee trees varied: under the canopy of F. vasta, F. sur, C. macrostachyus, S. abyssinica, $O$. capensis, P. adolfi-friederici, A. gummifera, P. oliveriana, $M$. ferruginea and $C$. africana we evidenced the highest colonization percentages. Coffee trees under $P$. reclinat $a$ and $P$. fulva showed significantly lower colonization percentages. At the other end of the spectrum, coffee trees under E. abyssinica and P. africana exhibited the lowest colonization percentages (Table 5). Arbuscular colonization ( $\mathrm{AC} \%$ ) is found to be greater in coffee trees than in their respective shade trees in almost all cases (Tables 4 and 6 ). However, in most cases vesicular colonization (VC \%) is larger in shade tree species than in the coffee trees that grow underneath. 


\begin{tabular}{|c|c|c|c|c|}
\hline Shade trees over coffee & HC (\%) & MHC (\%) & $\mathrm{AC}(\%)$ & VC (\%) \\
\hline Milletia ferruginea & $76 \pm 4^{b}$ & $5^{6} \pm 4^{a}$ & $13 \pm 2^{b c}$ & $5 \pm 2.0^{\text {def }}$ \\
\hline Schefflera abyssinica & $80 \pm 4^{a b}$ & $60 \pm 4^{a}$ & $15 \pm 2^{a b}$ & $4 \pm 0.3^{f}$ \\
\hline Croton macrostachyus & $83 \pm 5^{a b}$ & $6 I \pm 7^{a}$ & $\mathrm{I} 6 \pm 2^{\mathrm{ab}}$ & $2 \pm 0.6^{\mathrm{h}}$ \\
\hline Ficus vasta & $87 \pm 5^{a}$ & $64 \pm 9^{a}$ & $18 \pm 2^{a b}$ & $4 \pm 0.3^{f}$ \\
\hline Albizia gummifera & $7^{8} \pm 5^{a b}$ & $57 \pm 7^{\mathrm{ab}}$ & $19 \pm 2^{a}$ & $2 \pm 0.5^{h}$ \\
\hline Olea capensis & $80 \pm 4^{a b}$ & $57 \pm 3^{a}$ & $18 \pm 3^{a b}$ & $3 \pm 0.3^{g}$ \\
\hline Cordia africana & $73 \pm 3^{\mathrm{bc}}$ & $55 \pm 5^{a b}$ & $\mathrm{I} 2 \pm 2^{\mathrm{bc}}$ & $7 \pm 0.3^{\mathrm{cd}}$ \\
\hline Ehretia abyssinica & $5^{8} \pm 5^{c}$ & $39 \pm \mathrm{I}^{\mathrm{c}}$ & $8 \pm 2^{c}$ & $9 \pm 0.4^{b}$ \\
\hline Pouteria adolfi-friederici & $78 \pm 4^{\mathrm{ab}}$ & $57 \pm 5^{a}$ & $14 \pm 2^{b}$ & $6 \pm 0.6^{e}$ \\
\hline Pavetta oliveriana & $79 \pm 6^{\mathrm{ab}}$ & $5^{6} \pm 4^{a}$ & $\mathrm{I} 4 \pm 2^{\mathrm{b}}$ & $7 \pm 0.3^{d}$ \\
\hline Ficus sur & $7 \mathrm{I} \pm 3^{\mathrm{bc}}$ & $57 \pm 9^{a b}$ & $10 \pm 2^{b c}$ & $8 \pm 0.4^{c}$ \\
\hline Prunus africana & $59 \pm 6^{c}$ & $3^{6} \pm 7^{c}$ & $9 \pm 2^{c}$ & $7 \pm 0.3^{d}$ \\
\hline Phoenix reclinata & $67 \pm 4^{c}$ & $47 \pm 3^{b}$ & $9 \pm 2^{c}$ & $10 \pm 0.5^{a}$ \\
\hline Polyscias fulva & $60 \pm 4^{c}$ & $42 \pm 4^{b c}$ & $8 \pm \mathrm{I}^{\mathrm{c}}$ & $8 \pm 1.0^{b c d}$ \\
\hline
\end{tabular}

Table 5: Root colonization percentages of coffee plants under shade trees in Bonga, Ethiopia

(Where: $\mathrm{HC}$, hyphal colonization $=100[(G-p) / G] ; M H C$, mycorrhizal hyphal colonization $=100[(q+r+s+t) / G] ; A C$, arbuscular colonization $=100(q+s / G) ; V C$, vesicular colonization $=100(r+s / G)$. Where: $G=(p+q+r+s+t+u)$ intersections inspected, $p: n 0$ fungal structures, q: arbuscules, r: mycorrhizal vesides, s: arbuscules and mycorrhizal vesicles, t: mycorrhizal hyphae but no arbuscules or mycorrhizal vesicles and u: hyphae not seen to be connected to arbuscules or mycorrhizal vesides). 


\begin{tabular}{|c|c|c|c|c|c|c|c|c|c|c|c|c|c|c|}
\hline N & & $\begin{array}{l}\vec{H} \\
\hat{0}\end{array}$ & $\begin{array}{l}\text { బ̊ } \\
\text { స̦ } \\
0\end{array}$ & $\underset{ }{\stackrel{m}{0}}$ & $\underset{\hat{0}}{\stackrel{+}{*}}$ & $\begin{array}{l}\text { J } \\
\text { ○ }\end{array}$ & $\begin{array}{l}\text { ĩ } \\
\text { ì }\end{array}$ & $\begin{array}{c}\infty \\
\infty \\
\infty \\
i\end{array}$ & 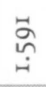 & 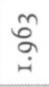 & $\begin{array}{c}\hat{n} \\
\text { ஸे } \\
0\end{array}$ & $\begin{array}{c}\stackrel{\Omega}{n} \\
\text { in }\end{array}$ & $\begin{array}{l}\text { ô } \\
\text { ¿ } \\
\end{array}$ & $\stackrel{n}{n}$ \\
\hline$\sum_{\Sigma}^{\infty 00}$ & $\frac{n}{i}$ & $\stackrel{\dot{m}}{i}$ & ఫa & $\begin{array}{l}n \\
\text { in }\end{array}$ & 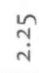 & $\stackrel{y}{\mathfrak{m}}$ & $\stackrel{n}{\tilde{n}}$ & $\begin{array}{l}\hat{\kappa} \\
\hat{i}\end{array}$ & $\hat{n}$ & $\stackrel{\infty}{\stackrel{\infty}{\Lambda}}$ & $\stackrel{m}{m}$ & $\stackrel{9}{i}$ & $\stackrel{n}{n}$ & $\stackrel{\circ}{i}$ \\
\hline తే & ‡ั & $\begin{array}{l}\hat{n} \\
\dot{m}\end{array}$ & 广̊ & $\underset{\mathfrak{n}}{\dot{m}}$ & $\stackrel{\tilde{n}}{\stackrel{n}{n}}$ & $\begin{array}{l}\infty \\
\text { ம } \\
\text { ம் }\end{array}$ & $\begin{array}{l}m \\
\dot{a}\end{array}$ & $\begin{array}{l}\infty \\
\dot{0} \\
\dot{a}\end{array}$ & i̊ & $\stackrel{\text { Yे }}{r}$ & $\underset{0}{\stackrel{0}{0}}$ & $\stackrel{N}{N}$ & $\begin{array}{l}\tilde{n} \\
\dot{n}\end{array}$ & $\stackrel{\sim}{\sim}$ \\
\hline 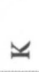 & م̂ & $\stackrel{\circ}{\circ}$ & $\stackrel{2}{3}$ & $\underset{7}{7}$ & $\begin{array}{l}\tilde{n} \\
\vdots \\
0\end{array}$ & $\stackrel{\circ}{\stackrel{0}{0}}$ & $\stackrel{\text { N }}{0}$ & $\stackrel{\overrightarrow{0}}{\circ}$ & $\begin{array}{l}\infty \\
0 \\
0\end{array}$ & $\begin{array}{l}\circ \\
0 \\
0\end{array}$ & $\stackrel{n}{n}$ & $\stackrel{ \pm}{ \pm}$ & స̃ & $\hat{\sigma}$ \\
\hline$\ddot{z}$ & సี & $\stackrel{9}{0}$ & $\stackrel{n}{0}$ & : & $\stackrel{n}{0}$ & ț & Ț & $\stackrel{+}{+}$ & $\stackrel{\infty}{\infty}$ & $\stackrel{\mathscr{0}}{0}$ & $\overrightarrow{7}$ & $\begin{array}{l}\text { กิ } \\
\text { ก }\end{array}$ & $\stackrel{\circ}{\circ}$ & $\stackrel{n}{0}$ \\
\hline પ્ઞુ & กิ & $\begin{array}{l}\text { กิ } \\
\text { ஸे }\end{array}$ & $\begin{array}{l}\text { กุ } \\
\text { ปn }\end{array}$ & ఝొ & $\stackrel{\sim}{\sim}$ & 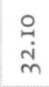 & 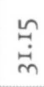 & $\begin{array}{l}\text { थ́ } \\
\stackrel{0}{n}\end{array}$ & $\begin{array}{l}\text { ڤூ } \\
\dot{N}\end{array}$ & $\begin{array}{l}\text { n } \\
\stackrel{\circ}{m}\end{array}$ & 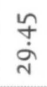 & $\begin{array}{l}\text { ヘ } \\
\text { ஸे }\end{array}$ & $\begin{array}{l}\infty \\
\infty \\
\text { ஸे }\end{array}$ & $\begin{array}{l}\mathfrak{n} \\
\stackrel{n}{n} \\
m\end{array}$ \\
\hline U & $\begin{array}{l}\text { gे } \\
\text { ó }\end{array}$ & $\begin{array}{l}\text { ñ } \\
0 \\
\dot{0}\end{array}$ & $\begin{array}{l}\hat{N} \\
\text { ọ } \\
\dot{0}\end{array}$ & $\begin{array}{l}\text { స్ } \\
\dot{0} \\
\dot{0}\end{array}$ & $\begin{array}{l}\text { ㄱ. } \\
\text { ơ } \\
\dot{0}\end{array}$ & $\begin{array}{l}0 \\
\text { I } \\
\dot{0}\end{array}$ & $\begin{array}{l}\text { ¿े } \\
0 \\
\dot{0}\end{array}$ & $\begin{array}{l}\vec{\circ} \\
\circ \\
0\end{array}$ & 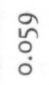 & $\begin{array}{l}\mathcal{N} \\
\infty \\
0 \\
0 \\
\dot{0}\end{array}$ & $\begin{array}{l}\text { ஸै } \\
\text { ○े }\end{array}$ & $\begin{array}{l}\text { กิ } \\
\text { ○ }\end{array}$ & \begin{tabular}{l}
$\circ$ \\
\multirow{1}{0}{} \\
$\dot{0}$
\end{tabular} & $\begin{array}{l}\circ \\
0 \\
0\end{array}$ \\
\hline$A$ & $\begin{array}{l}\circ \\
\dot{m}\end{array}$ & $\stackrel{m}{i}$ & 乞̊ & $\vec{a}$ & $\stackrel{\sim}{\stackrel{n}{\sim}}$ & 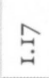 & 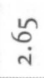 & $\begin{array}{l}\stackrel{\circ}{\circ} \\
\dot{f}\end{array}$ & $\stackrel{\stackrel{\leftrightarrow}{+}}{\text { t }}$ & $\stackrel{n}{i}$ & N & $\frac{\stackrel{+}{+}}{\dot{m}}$ & $\begin{array}{l}0 \\
\text { i }\end{array}$ & $\begin{array}{l}\tilde{o} \\
\dot{n}\end{array}$ \\
\hline Z & 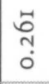 & 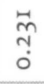 & $\stackrel{\circ}{\circ}$ & 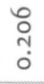 & $\begin{array}{l}\mathfrak{n} \\
\mathfrak{n} \\
0\end{array}$ & 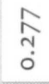 & 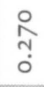 & $\stackrel{\hat{n}}{\mathfrak{n}}$ & 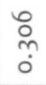 & $\stackrel{\substack{+\dot{m}}}{\stackrel{\infty}{0}}$ & $\begin{array}{l}\text { స̆ } \\
\text { o }\end{array}$ & $\begin{array}{l}\text { సิ } \\
\text { ○े }\end{array}$ & $\underset{\mathfrak{n}}{\mathfrak{O}}$ & 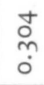 \\
\hline$\sum_{0}$ & $\begin{array}{l}\stackrel{0}{0} \\
\grave{i}\end{array}$ & $\underset{i}{\vec{i}}$ & \& & 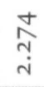 & $\underset{i}{i}$ & $\begin{array}{l}\text { ¿ } \\
\text { i }\end{array}$ & $\stackrel{m}{\tilde{n}}$ & $\begin{array}{l}\infty \\
\stackrel{+}{+} \\
\dot{m}\end{array}$ & $\begin{array}{l}\tilde{N} \\
\tilde{N}\end{array}$ & $\begin{array}{l}\hat{N} \\
\hat{i}\end{array}$ & 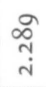 & $\begin{array}{l}\stackrel{+}{6} \\
\text { i }\end{array}$ & $\begin{array}{c}\mathbb{J} \\
\text { స } \\
\text { j }\end{array}$ & 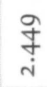 \\
\hline 䆓 & $\stackrel{\circ}{\circ}$ & $\stackrel{\circ}{\dot{j}}$ & $\stackrel{\circ}{\stackrel{ }{r}}$ & 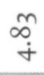 & $\stackrel{\Re}{\dot{f}}$ & $\stackrel{n}{\dot{q}}$ & $\stackrel{n}{\hat{n}}$ & $\stackrel{\stackrel{ }{ヘ}}{\dot{n}}$ & 올 & กั & $\begin{array}{l}\stackrel{n}{ก} \\
\stackrel{n}{n}\end{array}$ & $\stackrel{\infty}{m}$ & $\stackrel{\text { そ }}{+}$ & $\begin{array}{l}\tilde{n} \\
\dot{n}\end{array}$ \\
\hline $\begin{array}{l}\mathscr{\mathscr { U }} \\
\text { के }\end{array}$ & 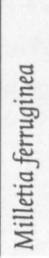 & 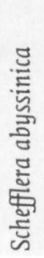 & 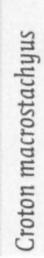 & 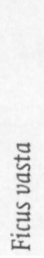 & 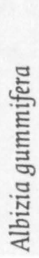 & 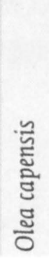 & 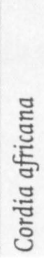 & 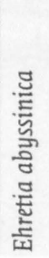 & 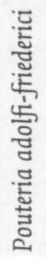 & 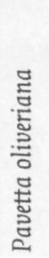 & 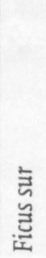 & 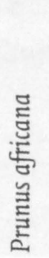 & 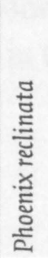 & 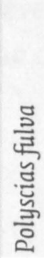 \\
\hline
\end{tabular}

Table 6: Physico-chemical characteristics of rhizosphere soil under each shade tree species in Bonga, Ethiopia Units used: $\mathrm{pH}\left(\mathrm{H}_{2} \mathrm{O}, 1: 2.5\right)$; $\mathrm{EC}$ in ds/m; $\mathrm{Na}, \mathrm{K}, \mathrm{Ca}, \mathrm{Mg}$ and $\mathrm{CEC}$ in $\mathrm{Cmol}(+) / \mathrm{kg} ; \mathrm{TN}$ and $\mathrm{OM}$ in \%; AuP and $\mathrm{Zn}$ in ppm 


\section{Soil characteristics and mycorrhization (Tables 6 and 7)}

Spore densities, HC, MHC and AC were inversely correlated with OM, total N, available $\mathrm{P}, \mathrm{EC}$ and $\mathrm{Zn}$ (Table 7.a). Only $\mathrm{HC} \%$ was negatively correlated with $\mathrm{pH}(\mathrm{r}=$ 0.540 ), whereas $\mathrm{VC}$ was not significantly correlated with any of the soil characteristics tested. A similar correlation trend was observed between coffee trees and soil characteristics, except for $\mathrm{AC}$ versus $\mathrm{OM}$ (Table 7.b). 


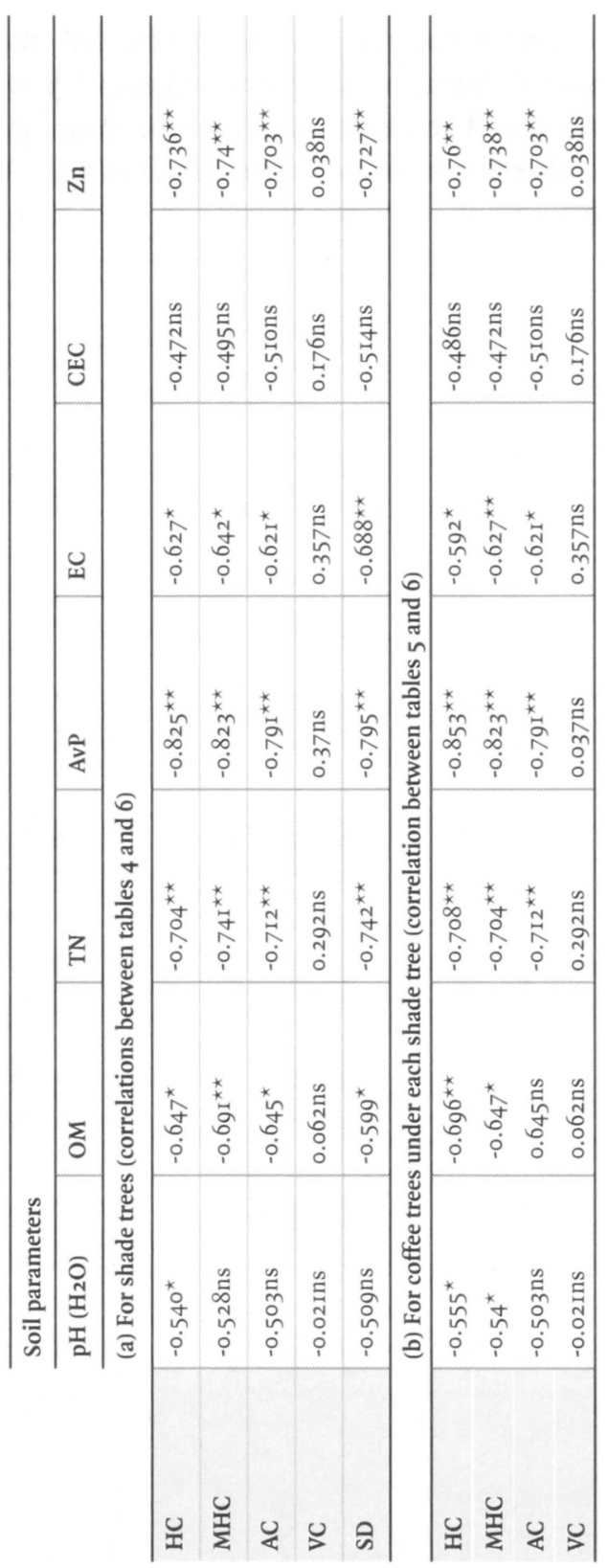

Table 7: Correlation between soil parameters and AM fungi of shade trees and the respective coffee trees underneath (Where: **-Correlation is significant at the 0.01 level; * - correlation is significant at the 0.05 level. ns, non significant at 0.05 level; SD, Spore density; $H C$, hyphal colonization $=100[(G-p) \mid G] ; M H C$, mycorrhizal hyphal colonization $=100[(q+r+s+t) \mid G]$; $A C$, arbuscular colonization $=100(q+s / G) ; V C$, vesicular colonization $=100(r+s / G)$. Where: $G=(p+q+r+s+t+u)$ intersections inspected. ( $p, q, r, s, t \& u$ are same as in the above tables)). 


\section{Discussion}

Four genera totaling 9 species of mycorrhizae were recovered from soils sampled from Bonga natural coffee forest, Ethiopia. Glomus was the dominant genus in all soils under each tree species both in terms of species diversity and spore density (Tables 2 and 3). This is in line with findings not only from dry afromontane forests of Ethiopia (Tesfaye et al., 2003b) and Bonga natural coffee forest (Muleta et al., 2007), but also from the tropical rain forest of Xishuangbanna, Yunnan, China (Zhao et al., 200I), tropical rain forest in Mexico (Guadarrama and Alvarez-Sanchez 1999), and arid and semi-arid lands of north Jordan (Mohammad et al., 2003).

This dominance of Glomus could be attributed to several factors. In the present study, it is observed that the acidic nature of the rhizosphere soil may have favoured this genus (Table 6). As we indicated earlier, Glomus species (with the exception of Glomus mosseae) have been found to be distinctly acid-tolerant (Mosse, 1972; 1973).

The least-occurring spore type, Acaulospora, was absent under S. abyssinica, C. macrostachyus, F. vasta, $O$. capensis and P. reclinata and when present was only evidenced at low spore densities (Table 3). The reasons for the low occurrence of this spore type are unclear. Low figures may be influenced by host types as a number of studies indicate that host-plant preferences exist (Halgason et al., 2002; Vandenkoornhuyse et al., 2003; Johnson et al., 2003). This could either be due to differences in root anatomy or exudates from plant roots (Gamalero et al. 2004; Norman et al., 1996). Cardoso et al. (2003) also indicated that spore production or colonization could be influenced by the length of plant roots.

However, the number of species (especially Glomus) isolated as spores from any forest may be lower than that of pot cultures (Brundrett et al., 1999). The number of species recovered in this study might be underestimated due to the fact that some species may not produce their spores in forest soil.

F. vasta, C. macrostachyus and S. abyssinica harboured relatively greater number of spores than other hosts. Fewest spores were encountered under E. abyssinica, P. africana and P. fulva. Spore numbers were negatively associated with available $\mathrm{P}$, total $\mathrm{N}$, and $\mathrm{Zn}$ in soil, and variably associated with organic matter, EC and CEC. Because mycorrhizae help to increase uptake of minerals in plants, the levels of $\mathrm{P}$ and $\mathrm{Zn}$ in the plant may regulate root colonization and spore formation (Smith and Read, 1997). It has been shown that high levels of phosphorus in soil and plant are able to inhibit mycorrhiza formation (Douds and Schenck, I990) and influence the diversity of AM fungi in field soils (Cuenca and Menses, r996).

Generally, a larger number of mycorrhizal spores were extracted under each tree in this study than in other studies. In this work, the lowest average number of AM fungi spores extracted (578/100 g) was larger than those presented by Yonas (2005) who reported 57.9 spores per $100 \mathrm{~g}$ dry soil under Acacia polyacantha in a dry savannah woodland ecosystem. Similarly, Shasho (2002) reported more than 300 spores per $100 \mathrm{~g}$ soil beneath Erythrina brucei from a highland woodland ecosystem. These differences in spore density may be due to variations in environment, host trees and edaphic factors between study places. The higher spore number in this coffee forest may be due to the low level 
of soil disturbance where almost no tillage is practiced in the forest. Similar results were obtained in Brazil by Cardoso et al. (2003).

The pattern of root colonization was also found to vary among shade trees (even between members of the same family). The variations in some species with relation to the degree of colonization and presence/absence of AM fungi in the same or different collecting places indicate that environmental factors influence the presence or absence of mycorrhizae and their colonization level (Alexander, I989). Similarly, St. John (I980) indicated such a variation of root colonization to occur at genus and family levels.

A direct relationship was found between colonization percentage and spore density in the soil in each tree species. Jasper et al. (1993) and Frank and Morton (1994) similarly observed sporulation to be positively correlated with mycorrhizal colonization. Therefore, this may indicate that most of the spores in this study are colonizing ones.

Generally, the coffee tree roots were more heavily colonized by arbuscules (AC\%) than their respective shade trees (significant difference at the 0.05 level). General hyphal colonization ( $\mathrm{HC} \%$ ) and mycorrhizal hyphal colonization ( $\mathrm{MHC} \%$ ) were also seen to be slightly higher in coffee trees than in their respective shade trees. However, these differences were only statistically significant in Pouteria adolf-friederici (in case of HC\%) and Cordia africana (in case of $\mathrm{MHC} \%$ ).

The reasons for the relatively higher coffee root colonization are unclear. However, the implications may be important. Greater colonization in coffee may indicate lower relative available $\mathrm{P}, \mathrm{Zn}$ and $\mathrm{N}$ for coffee than for its companions.

It is not clear why AC\% seems to be higher in coffee roots than in tree roots, whereas VC\% is lower in coffee than in shade trees. However, this may be because coffee tree roots are more colonized by non-vesicle forming mycorrhizal species, or it might be explained by the fact that coffee trees need to have nutrients in the short term rather than to store them in vesicles (nutrient storage sites). This would support the idea that transfer of assimilates from one plant to another is facilitated more through arbuscules than by vesicles (Grime et al., 1987).

Spore density and proportional colonization (for both coffee and shade trees) were negatively correlated with organic carbon, total $\mathrm{N}$, available $\mathrm{P}, \mathrm{EC}$ and $\mathrm{Zn}$ (Tables 7.a and 7.b). However, the correlation between arbuscular colonization for coffee (AC\%) and organic carbon was positive but not significant at 0.05 level (Table 7.b). Abiotic factors had minimal influence on mycorrhizal colonization variation (Bohrer et al., 2004).

The present result showed that correlations were very significant between edaphic factors and mycorrhizal colonization except for vesicular colonization (VC\%) (Tables 7.a and 7.b). This strongly supports the idea that both climatic and edaphic factors could influence the mycorrhizal colonization (Staddon et al., 2003). AM fungi could enhance plant uptake of $\mathrm{P}$ and other nutrients, especially in nutrient-deficient environments (Smith and Read, 1997). Similarly, Cardoso et al. (2003) postulated that greater mycorrhizal activity in the deeper soil layers may be important in making more P available to the plant and thus increase the efficiency of nutrient recycling processes in agroforestry systems. 
This may again be related to root architecture. Nutrient availability had a much stronger effect on root architecture than arbuscular mycorrhiza (Cruz et al., 2004). Mycorrhizae increase the uptake capacity of the active root zone. However, it is wellknown that high soil P suppresses mycorrhizal activity mainly through its effect on the P concentration in plants (Bowen 1987; Menge et al., 1978).

The importance of mycorrhizae to coffee has been reported by several investigators indicating that coffee plants will be heavily mycorrhized under natural conditions. Benefits include enhanced growth and increased $\mathrm{P}$ and $\mathrm{Zn}$ uptake by young coffee seedlings in nursery conditions (Lopes et al., 1985; Siqueira et al., 1998); enhanced tolerance to nematodes (Vaast et al., 1998) and increased survival of coffee plants after field transplanting and in agroforestry systems (Vaast and Zasoski, 1992). Agroforestry systems can increase soil nutrient availability and accelerate $P$ cycling because the deeper tree roots retrieve nutrients from lower soil horizons (Young, 1997), enhance the chemical and physical quality of soils and increase soil microbial activity (Cooper et al., 1996).

\section{Conclusions}

A diverse population of mycorrhizae was observed as spores in a coffee forest in Bonga, Ethiopia. Presence of spores was associated with high levels of mycorrhizae on the roots of both the forest species and coffee plants growing under these forest species. Mycorrhizal colonization in coffee was higher than in the companion forest species, indicating that mycorrhizae may be more helpful for coffee than for the companions. Colonization of both coffee and shade trees was correlated with several edaphic characters of the soil in which they were growing. These observations indicate that management of coffee groves should take into account the impact of various practices on the mycorrhizae, and try to maintain high levels of colonization. The specific management practices needed to maintain mycorrhizae in this system are unclear, but absence of soil disturbance and proper management of litter fall (OM) may be important, and are worth further investigation.

\section{Acknowledgements}

The authors would like to acknowledge Dr. Diriba Muleta because he provided relevant articles and unreserved professional advice, and Dr. Seyoum Leta, for his help in taking microphotographs of spores and colonized roots using a cci2-model camera mounted on an Olympus Bx 5 I microscope which was connected to the analySIS ${ }^{\circledR}$ Soft Imaging Systems $\mathrm{GmbH}$ version 3.2 analysis program. We are very grateful to Ato Endawoke Asegid for preparing a map of the study area using GIS. This study was financially supported by Microbial Inputs into Agroforestry, a project between the Swedish University of Agricultural Sciences (SLU), Sweden and Addis Ababa University, Ethiopia, funded by SIDA/SAREC. 


\section{References}

Aga, E., Bryngelsson, T., Bekele, E. \& Salomon, B. (2003). Genetic diversity of forest arabica coffee (Coffea arabica L.) in Ethiopia as revealed by random amplified polymorphic DNA (RAPD) analysis. Hereditas, 138 : 36-46.

Alexander, I.J. (1989). Mycorrhizas in tropical forests. In: J. Proctor (ed.), Mineral nutrients in tropical forest and savannah ecosystems, pp.169-188. Oxford: Blackwell.

Bohrer, K.E, Friese, C.F \& Amon, J.P.O. (2004). Seasonal dynamics of arbuscular Mycorrhizal fungi in differing wetland habitats. Mycorrhiza, I4: 329-337.

Bourou, S., Diouf, M. \& Van Damme, P. (2010). Tamarind (Tamarindus indica L.) parkland mycorrhizal diversity within three agro-ecological zones of Senegal. Fruits, 65 (6), 377-385.

Bourou, S., Ndiaye, F., Diouf, M. \& Van Damme, P. (20II). Effets de l'inoculation mycorhizienne sur le comportement agro-physiologique des écotypes du tamarinier (Tamarindus indica L.) au Sénégal. Journal Applied Biosciences, 46, 3093-3102.

Bowen, G.D. (1987). The biology and physiology of infection and its development. In: G.R. Safir (ed.), Ecophysiology of VA mycorrhizal plants, pp. 25-27. Boca Raton, Fla: CRC Press.

Bremner, J.M. \& Mulvaney, C.S. (1982). Nitrogen Total. In: A.L. Page (ed.), Methods of soil analysis. Part 2. Chemical and Microbiological properties, pp. 595-64I. SSSA, Madison: Wisconsin.

Brundrett, M.C., Piche, Y. \& Peterson, L. (1994). A new method for observing the morphology of VAM. Can. J. Bot., 62: 2128-2134.

Brundrett, M.C., Bougher, N., Dell, B., Grove, T. \& Malajczuk, N. (1996). Working with mycorrhizas in forestry and agriculture. ACIAR Monograph 32. Australian Center for International Agricultural Research, Canberra.

Brundrett, M.C., Abbott, L.K. \& Jasper, D.A. (1999). Glomalean Mycorrhizal fungi from tropical Australia I. Comparison of the effectiveness and specificity of different isolation procedures. Mycorrhiza, 8: 305-314.

Cardoso, I.M., Boddington, C., Janssen, B.H., Oenema, O., \& Kuyper, T.W. (2003). Distribution of mycorrhizal fungal spores in soils under agroforestry and monocultural coffee systems in Brazil. Agrofor Syst., 58:33-43.

Cooper, P.J.M., Leakey, R.R.B., Rao, M.R. \& Reynolds, L. (1996). Agroforestery and the mitigation of land degradation in the humid and sub-humid tropics of Africa. Exp. Agric., 32: 235-290.

Cruz, C., Green, J.J. \& Watson, C.A. (2004). Functional aspects of root architecture and mycorrhizal inoculation with respect to nutrient uptake capacity. Mycorrhiza, I4: 177-184.

CSA (Central Statistical Authority) (2002). Annual Statistical Abstract. Addis Ababa, Ethiopia.

Cuenca, G. \& Meneses, E. (1996). Diversity patterns of arbuscular mycorrhizal fungi associated with cacao in Venezuela. Plant Soil, $183: 315-322$.

Daba, W. (2002). Forests and forestry's role in food security: The forgotten intervention in Food security efforts. In: T. Demel \& Y. Yonas (eds.), "Forest and environment," Proceedings of the fourth Annual conference forestry society of Ethiopia, pp. 32-37, Addis Ababa, January 14-15.

Demel, T. \& Tegineh, A. (1991). Traditional Tree Crop Based Agroforestry in Coffee Producing Areas of Harerge, Eastern Ethiopia. Agrof. Syst., I6: 253-267.

Douds, D.D. \& Schenck, N.C. (1990). Relationship of colonization and sporulation by VA mycorrhizal fungi to plant nutrient and carbohydrate contents. New Phytol., Ir6: 62I-627.

Douds, D.D., Gadkar, V. \& Adholeya, A. (2000). Mass production of VAM fungus biofertilizer. In: K.G. Mukerji \& P. Chamola (eds.), proceedings of mycorrhizal biology, pp.197-215, TERIIN. ORG.

Ethiopian Institute of Agricultural Research, (EIAR) (1996). Recommended Production Technologies for Coffee and Associated Crops, pp. 3-8, Addis Ababa, Ethiopia.

FAO. (1968). World Coffee Survey. FAO Agricultural studies. No. 76, pp. I-2I, Rome.

Francis, R. \& Read, D.J. (1994). The contributions of mycorrhizal fungi to the Determination of plant community structure. In: A.D. Robson, L.K. Abbott \& N. Malajczuk (eds.), Management of mycorrhizas in Agriculture and Forestry, pp. II-26, London: Kluwer Academic publishers. 
Frank, M. \& Morton, B. (1994). On to genetic comparison of arbuscular mycorrhizal fungi Scutellospora heterogama and Scutellospora pellucida: revision of taxonomic character, concepts, species descriptions and phyolgenetic hypothesis. Can. J. Bot., 72: 122-134.

Frioni, L., Minasian, H. \& Volfovicz, R., (1999). Arbuscular mycorthizal and ectomycorrhizal in nature tree legumes in Uruguay. For. Ecol. Manag., I15: 41-47.

Gamalero, E., Trotta, A., Massa, N., Copetta, A., Martinotti, MG. \& Berta, G. (2004). Impact of two fluorescent pseudomonads and an arbuscular mycorrhizal fungus on tomato plant growth, root architecture and P acquisition. Mycorrhiza, 14: 185-192.

Grime, J.P., Mackey, J.M., Miller, S.H. \& Read, D.J. (1987). Floristic diversity in a model system using experimental microcosm. Nature, 328: 420-422.

Guadarrama, P. \& Alvaarez-Sanchez, F.J. (1999). Abundance of Arbuscular Mycorrhizal Fungi spores in different environments in a tropical rain forest, Veracruz, Mexico. Mycorrhiza, 8: 267-270.

Van der Heijden, M.G.A., Boller, T.,Wiemken, A. \& Sanders, J.A. (1998). Different Arbuscular Mycorrhizal fungal species are potential determinants of plant community structure. Ecology, 79 (6): 2082-2091.

Helgason, T., Merryweather, JW, Denison, J., Wilson, P., Young, JPW., Fitter, AH. (2002). Selectivity and functional diversity in arbuscular mycorrhizas of co-occurring fungi and plants from a temperate deciduous woodland. J Ecol., 90: 371-384.

International Coffee Organization (ICO). "Botanical Aspects". http://www.ico.org/botanical.asp. Retrieved on 2009-07-03.

INVAM (2004). Identification of AMF to genus level. http://imvam.caf.wvu.edu.

Jasper, D.A., Abott, L.K. \& Robson, A.D. (1993). The survival of infective hyphae of vesicular- arbuscular mycorrhizal fungi in dry soil: an interaction with sporulation. New Phytol., 124: 473-479.

Johnson, D., Vandenkoornhuyse, PJ., Leake, JR., Gilbert, L., Booth, RE., Grime, JP., Young, PW., Read, DJ. (2003). Plant communities affect arbuscular mycorrhizal fungal diversity and community composition in grassland microcosms. New Phytol., i61: 503-515.

Kormanik, P.P. \& McGraw, A.C. (1982). Quantification of vesicular-arbuscular mycorrhizae in plant roots. In: N.C. Schenck (ed.), Methods and principles of mycorrhizal research, Minnesota: Am. Phytopathol. Soc.

Kubota, M. \& Hyakumachi, M. (2004). Morphology and colonization preference of arbuscular mycorrhizal fungi in Clethra barbinervis, Cucumis sativus, and Lycopersicon esculentum. Mycoscience, 45: 206-213.

Lopes, E.S., Dias, R., Toledo, S.V., Hiroce, R. \& Oliviera, E. (1985). Nutrient uptake by coffee inoculated with Gigaspora margarita. In: Proceedings: 6 North Amer. Conf. on Mycorrhizae, p.156, Oregon State University, Corvallis, OR, 1984 .

McGonigle, T. P., Miller, M. H., Evans, D.G., Fairchild, D.G. \& Swann, J.A. (1990). A new method which gives an objective measure of colonization of roots by vesicular-arbuscular mycorrhizal fungi. The New Phytologist, II5: 495-501.

Menge, J.A., Steirle, D., Bagyaraj, D.J., Johnson, E.L.V. \& Platt, R.G. (1978). Phosphorus concentration in plants responsible for inhibition of mycorrhizal infection. New Phytol., 80: 575-578.

Merryweather, J. (2004). Techniques in Arbuscular Mycorrhizal Research. Laboratory manual. University of York. Below ground research team.

Mesfin, A. (1991). General Introduction: An overview on the status of coffee, tea and spice in Ethiopia. In: Proceedings of the First Workshop on Production Constraints Assessment of Coffee, Tea and Spices, Jimma, Ethiopia, September 15-17.

Million, Y. (2002). The effect of indigenous Arbuscular mycorrhizal fungi (AMF) on growth and nitrogen fixation of two Acacea trees of Ethiopia, MSC. (Biology) thesis submitted to Addis Ababa University, Ethiopia.

Ministry of Agriculture, (MOA). (1998). Agroecological zones of Ethiopia: Natural resource management and regulation department, Ministry of Agriculture, Addis Ababa, Ethiopia.

Mohammad, M.J., Hamad, S.R.\& Malkawi, H.I. (2003). Population of arbuscular Mycorrhizal fungi in semiarid environment of Jordan as influenced by biotic and abiotic factors. J. Arid Environ., 53:409-417. 
Mosse, B. (1972). The influence of soil type and Endogone strain on the growth of Mycorrhizal plants in phosphatedeficient soils. Revue d'Ecologie et de Biologie du Sol, 9: 529-539.

(1973). Advances in the study of vesicular-arbuscular mycorrhiza. Annual Review of Phytopathology, II: I71-196.

Muleta, D., Assefa, F., Nemomissa, S., \& Granhall, U. (2007). Composition of coffee shade tree species and density of indigenous arbuscular mycorrhizal fungi (AMF) spores in Bonga natural coffee forest, southwestern Ethiopia. For. Eco. Manag., 241:145-154.

Norman, J.R., Atkinson, D. \& Hooker, J.E. (1996). Arbuscular mycorrhizal fungal induced-alteration to root architecture in strawberry and induced resistance to the root pathogen Phytophtora fragariae. Plant and Soil, I85: 191-198.

Paulose, D. \& Zebene, M. (1994). Ecology and soils of major coffee growing regions of Ethiopia. In: D. Paulose (ed.), Mineral Fertilization for Coffee in Ethiopia, pp. I-38. Addis Ababa Ethiopia: IAR.

Paulose, D. \& Demel, T. (2000). Coffee production systems in Ethiopia. In: Proceedings of the workshop on control of coffee berry disease (CBD) in Ethiopia, pp. 99-107, Addis Ababa, Ethiopia, 3-15 August 1999.

Rivera, R.F., Fernández, A., Hernández, J.R. \& Fernández, T.R. (2003). El manejo efectivo de la simbiosis micorrizica, una vi'a hacia la agricultora sostenible: estudio de caso. El caribe: Ciudad de La Habana.

Shasho, M. (2002). Dominant Rhizobacteria and Arbuscular Mycorrhizal Fungi (AMF) on the growth and N-fixation of Erythrina brucei (Korch) MSc. (Biology) thesis submitted to Addis Ababa University, Ethiopia.

Sieverding, E. (199I). Vesicular-arbuscular mycorrhiza management in tropical agrosystems. Federal Repuplic of Germany: Eschborn Technical Cooperation, ISBN 3-88085-462.

Siqueira, J.O., Saggin-Junior, O.J., Flores-Aylas, W.W. \& Guimaraes, T.G. (1998). Arbuscular Mycorrhiza Inoculation and Superphosphate Application Influence Plant development and yield of coffee in Brazil. Mycorrhiza, 7: 293-300.

Staddon, P.L., Thompson, K., Jakobsen, I., Greme, J.P., Askew, A.P. \& Fitter, A.H. (2003). Mycorrhizal fungal abundance is affected by long-term climatic manipulations in the field. Global change Biol., 9: 186-194.

St. John, T.V. (1980). A survey of mycorrhizal infection in an Amazonian rain forest. Acta Amazonica, 10: 527533.

Tan, K. H. (1996). Soil sampling, preparation and analysis. New York: Marcel Dekker, Inc.

Taye K. and Burkhard M. J. (2OII). Plant composition and growth of wild Coffea arabica: Implications for management and conservation of natural forest resources. Int. J. Biodvers. Conserv. 3(4): I3 I-I4I.

Tefestewold, B. (1995). Studies on Colletotrichum population of Coffea arabica L. in Ethiopia and evaluation of the reactions of coffee germplasms. Doctoral Dissertation. Hohen Landwirtschaftlichen Fakultat der Rheinschen Friedrich-Wilelms-Universität Zu Bonn. p. 23.

Tesfaye, W., Kottke, I., Demel, T. \& Oberwinkler, F. (2003a). Mycorrhizal status of Indigenous trees in dry Afromontane forests of Ethiopia. For. Ecol. Manag., 179: 387-399.

(2003b). Molocular diversity of arbuscular Mycorrhizal fungi in Prunus africana, an endangered medicinal tree species in dry afromontane forests of Ethiopia. New Phytol., I61:517-528.

Vaast, P.H. \& Zasoski, R.J. (1992). Effects of VA-mycorhizae and nitrogen sources on rhizosphere soil characteristics, growth and nutrient acquisition of coffee seedlings (Coffea arabica L.). Plant Soil, I47: 31-39.

Vaast, P.H., Caswell-Chen, E.P. \& Zasoski, R.J. (1998). Influences of a Root-Liosion Nematode, Pratylenchus coffeae, and two Arbuscular mycorrhizal Fungi, Acaulospora mellea and Glomus clarum on coffee (Coffea arabica L.). Biol. Fertil. Soils, 26:130-135.

Vandenkoornhuyse, P., Husband, R., Daniell, T.J., Watson, I.J., Duck, M., Fitter, A.H., Young, J.P.W. (2003). Co-exsisting grass species have distinctive arbuscular mycorrhizal communities. Mol Ecol., I2: 30853095 .

Van Ranst, E., Verloo, M., Demeyer, A. \& Pauwels, J.M. (1999). Manual for the soil chemistry and fertility laboratory: analytical methods for soils and plants, equipments and management of consumables. University of Gent, Belgium. 
Van Reeuwijk, L.P. (1993). Procedurıs for soil analysis. $4^{\text {th }}$ ed. Netherlands: International soil reference and Information Center.

Wolf, H. (1999). Methods and Strategies for the Conservation of Forest Genetic Resources. In: S. Edwards, D. Abebe, B. Taye \& G. Haase (eds.), Forest Genetic and Actions: proceedings of the national Forest Genetic Resources Conservation Strategy Development Work Shop, pp. 83-100, Institute of Biodiversity Conservation and Research, Addis Ababa, $21-22$ June 1999.

Yonas, Y. (2005). Symbiotic and Phenotypic characteristics of root nodule Bacteria and Arbuscular Mycorrhizal Fungi infecting Acacia polyacantha growing in Ghibe wooded grasslands. MSc. (Biology) thesis submitted to Addis Ababa University, Ethiopia.

Young, A. (1997). Agroforestry for soil Management. 2nd ed. Wallingford, UK: ICRAF and CAB International, p. 320 .

Zhao, Z., Xia, Y., Qin, X., Li, X., Cheng, L., Sha, T. \& Wang, G. (200I). Arbuscular Mycorrhizal status of plants and the spore density of Arbuscular Mycorrhizal fungi in the tropical rain forest of Xishuangbanna, southwest China. Mycorrhiza, II: 159-162. 